\title{
Preface to Volume 1 Issue No. 3
}

\author{
Gerasimos Rigatos - Pierluigi Siano
}

(C) Springer Science+Business Media Singapore 2015

In issue no. 3 emphasis is given again in robotic and electric power systems. Actually, in the area of autonomous robotic systems two significant control methods are presented. The first one is a nonlinear control approach for the steering of autonomous vehicles which is based on local linearization of the vehicle's dynamic or kinematic model and on the application of H-infinity control on the linearized equivalent model of the system. The second one is an adaptive control approach for autonomous vessels and submersibles which is based on global linearization of the vessel's dynamics with the use of differential flatness theory and on the development of an adaptive control scheme which assures stability without any need for prior knowledge of the system's dynamics. Moreover, in the area of electric power systems the issue provides significant results on optimal allocation and use of power storage devices in microgrids and on improved power management in buildings and in residences. Besides, it analyzes methods for the dynamic trading of electric power within an open energy market and under the presence of multiple energy providers. The trading of electric power takes place between distributed power suppliers on the one side and residential/industrial customers and owners of electric vehicles on the other side. It is shown how a balance in the electricity market can be reached through dynamic auction procedures.

In article A New Nonlinear H-infinity Feedback Control Approach to the Problem of Autonomous Robot Navigation by G. Rigatos and P. Siano a new control method is presented which is based on approximate linearization of the nonlinear system dynamics and on the application of a robust $\mathrm{H}$-infinity control scheme on the linearized equivalent model of the system. The computation of the feedback gain requires the solution of a Riccati equation at each iteration of the control algorithm. The stability of the control method is proven through Lyapunov analysis. Yet simple.
The proposed control method succeeds excellent results and achieves that the state variables of the control system will track precisely the reference setpoints. As test case the problem of control of an autonomous robotic vehicle is considered (unicycle robot). The robot is shown to track the reference path with remarkable accuracy and is also shown to complete efficiently the parallel parking task. This article has been approved by both Editors-in-Chief G. Rigatos and P. Siano.

In article Flatness-Based Adaptive Fuzzy Control of Autonomous Submarines by G. Rigatos and P. Siano a new method based on differential flatness theory and adaptive fuzzy control is proposed for MIMO nonlinear dynamical systems, having as particular application example the motion control of an autonomous submarine. The method exhibits specific advantages comparing to other adaptive fuzzy and neural control methods. These are summarized as follows: (i) it achieves excellent accuracy in the tracking of the reference setpoints. This is a confirmation of the method's stability analysis that results in an H-infinity tracking performance criterion and in an asymptotic stability condition. Moreover, it has been shown that the control input exhibits smooth variations. This is an advantage comparing, for instance, to adaptive fuzzy sliding-mode control since in the latter case there are abrupt control input variations. (ii) It outperforms all other adaptive neural/fuzzy control methods because it can be applied to a wider class of nonlinear dynamical systems. The method can be applied to any type of MIMO differentially flat systems that admit a transformation to the canonical Brunovsky form and does not require the systems' dynamic model to have a particular structure (e.g., affine-in-the-input, triangular or other). This article has been approved by both Editors-in-Chief G. Rigatos and P. Siano.

In article A Modified Genetic Algorithm for Optimal Allocation of Capacitor Banks in MV Distribution Networks by A. 
Augugliaro, L. Dusonchet, S. Favuzza, M. Ippolito, S. Mangione and E. Sanseverino a genetic algorithm is developed in which each individual is represented not by a string but by a matrix: in this way the entire population is represented by a 3-D matrix. According to the authors' analysis such a representation helps in solving more efficiently optimization problems with many discrete variables. As an application example, the authors consider the optimal allocation and sizing of distributed generation systems or the optimal compensation in a distribution system through the installation, in a prefixed number of nodes, of batteries and capacitors of variable size. In these cases, the unknowns of the optimization process are the nodes where the apparatus can be installed and their rated sizes can be chosen from a discrete set of values. The authors' approach enables to define new operators for the update of the chromosomes in the genetic algorithm and consequently results in better search in the parameters' space towards the optimal solution. This article has been approved by both Editors-in-Chief G. Rigatos and P. Siano.

In article Energy Semantic Network for Building Energy Management by H. Gabbar, A. Eldessouky and J. Runge an energy semantic network (ESN) is proposed for analyzing buildings' thermal performance (depending on parameters such as insulation materials, construction characteristics, and energy needs of residents). The developed ESN enables to study various schemes of energy supply and conversion as well as to evaluate different levels of power consumption in buildings. The ESN has a flexible structure which permits to modify, add, or delete data structures and classes associated with energy management of the building. In addition, the ESN's code is optimally designed so as to avoid computational burden during simulation and monitoring of the building's energy inflow and consumption. The proposed ESN is assessed through case studies about mid-size residences connected to different energy sources and partitioned in different thermal zones. This article has been approved by both Editors-in-Chief G. Rigatos and P. Siano.

In article A Novel Operating Strategy for Customer-Side Energy Storages in Presence of Dynamic Electricity Prices by E. Telaretti, E. Sanseverino, M. Ippolito, S. Favuzza and G. Zizzo a novel charging strategy is presented for the management of electric power storage systems in the case of hourly variation of electricity prices. The proposed power trading approach aims to maximize the profit of the storage owner (electricity customer) by determining an optimal charge/discharge schedule. The optimal operating schedule of storage devices is obtained by maximizing an objective function which corresponds to an elevated benefit for the power consumer. The model can be applied to several types of power storage devices although the article's simulations focus on lead-acid batteries. Test results show that the proposed power storage management can conditionally achieve the maximization of the profit for the power consumer. The results also show that pay-back of the investment for the purchase of power storage equipment can be succeeded after a couple of years and provided that lead-acid batteries are used. This article has been approved by both Editors-in-Chief G. Rigatos and P. Siano.

In article Impact of Incentive Mechanism on Demand Response Programs in Smart Microgrids with Electric Vehicles by H. Kumar Nunna, S. Battula, A. Sesetti and S. Doolla a new incentive mechanism called priority rewarding is proposed to manage the charging schedule of electric vehicles. The proposed vehicles' charging policy is based on short term electricity price forecasting and on bilateral contracts between the power consumers and distributed power suppliers. The electricity trading scheme is further supported by a second price auction mechanism. In order to prove the suitability of the proposed incentive mechanism with the DR strategy within a microgrid infrastructure, an agentbased energy trading scenario is developed using a Java agent programming environment. The developed agent system is applied to a case study model of a grid connected microgrid and the obtained results have shown that the proposed methodology can be successfully adopted in practice. This article has been approved by both Editors-in-Chief G. Rigatos and P. Siano.

In article Multi-agent System Design Principles for Resilient Coordination and Control of Future Power Systems by Amro M. Farid a set of multi-agent system design principles for robust coordination and control of future power systems is identified. It draws upon an axiomatic design for large flexible engineering systems model which was recently used in the development of robustness features. From this quantitative model, a set of design principles are easily distilled. The article also assesses the adherence of existing multiagent system implementations with respect to these design principles. The article has been approved by Editor-in-Chief P. Siano.

The articles which have been shortlisted for inclusion in this new issue of the Journal of Intelligent Industrial Systems are timely and novel and have been carefully evaluated through a meticulous review procedure. Through the aforementioned articles recent advancements in the area of industrial systems are reflected and the potential of this research area for further growth is demonstrated. Robotic systems, intelligent autonomous vehicles and smart electricity networks are fields exhibiting the need for innovation, knowhow transfer and application of advanced information technology methods. The issue has shown the improvement that is achieved in the functioning of such indus- 
trial systems by using elaborated control and decision making software and has pointed out key technologies that will steer the development of this area in the forthcoming years.
G. Rigatos

Unit of Industrial Automation Industrial Systems Institute, 26504 Rion Patras,

Greece

email: grigat@ieee.org

P. Siano

Via Giovanni Paolo II

132, 84084 Fisciano, SA, Italy

emial: psiano@unisa.it 